\title{
US intensity and blood-pressure effects on classical conditioning and sensitization in spinal cat
}

\author{
ALAN R. LIGHT and RUSSELL G. DURKOVIC \\ Department of Physiology, Upstate Medical Center, Syracuse, New York 13210
}

\begin{abstract}
Acute spinal cats were immobilized with lesions which produced either high or low mean arterial blood pressures. These animals were assigned to classical conditioning or sensitization paradigms in which the conditioned and unconditioned stimuli (CS and US) were shocks to two different cutaneous nerves of the left hindlimb. Conditioned and unconditioned responses were flexion reflex tensions recorded from the isolated tibialis anterior muscle of the same leg. Three different US intensities were employed for both conditioning and sensitization groups. While conditioning animals received paired stimuli, sensitization animals received either a pseudorandom or an alternating schedule of unpaired CS and US presentations. Spinal cats receiving US intensities which activated at least $\mathrm{A} \alpha$ and Ad cutaneous afferents showed greater flexion reflex facilitation during the conditioning paradigm than in the sensitization paradigm. The addition of $\mathbf{C}$ fiber activation to the Aad US produced no additional influence on net conditioning. A US intensity which activated only $\mathrm{A} \alpha$ fibers did not produce facilitation in either conditioning or sensitization paradigms. High- and low-blood-pressure groups behaved similarly, demonstrating the independence of spinal conditioning from this physiological factor over the range observed. No differences were observed between alternating and pseudorandom sensitization control groups.
\end{abstract}

Recent experiments have confirmed that the isolated spinal cord can exhibit classical conditioninglike behavior (Durkovic, 1975; Durkovic \& Light, 1975; Fitzgerald \& Thompson, 1967; Patterson, 1975, 1976; Patterson, Cegavske, \& Thompson, 1973). These findings are important because they indicate that the spinal cord may be useful as a simplified neural model for the neurophysiological study of classical conditioning phenomena. We have been trying to further simplify the spinal cord model by determining the minimal afferent inputs required to produce conditioned responding. A previous report (Durkovic \& Light, 1975) suggested that a US which activated at least $\mathrm{A} \alpha$ and $\mathrm{A} \delta$ cutaneous nerve fibers was necessary in order to produce classically conditioned facilitation of the flexion reflex. However, this study did not contain sensitization control animals. Thus the amount of facilitation specific to pairing of stimuli could not be determined.

The present study, then, was conducted for several purposes. The primary purpose was to determine the effects of US intensity during both conditioning and sensitization paradigms in order to determine the minimal US intensity for producing optimal conditioning, with the least obscuring sensitization effects.

This research was supported by a grant-in-aid from the Joint Awards Council/University Awards Committee of State University of New York Research Foundation and by National Science Foundation Grant BNS75-16747. A. R. Light was supported by Public Health Service Predoctoral Training Grant 5T01GM1006.
Another purpose was to investigate the effects of high and low mean arterial blood pressure on conditioning and sensitization in the spinal cat. Previous experiments in our laboratory have been conducted on cats rendered unconscious by anemic decapitation. This procedure produced animals with lower than normal blood pressure. We hypothesized that animals with higher mean blood pressures, found in animals rendered unconscious with midbrain transections, might exhibit less response variability. Such decreased variability could have resulted in a greater distinction between conditioning and sensitization animals and, thus, increase the efficiency of subsequent neurophysiological investigations of this phenomenon.

Also incorporated into the present experimental design was a comparison of two types of sensitization control paradigms. Previous sensitization controls in our laboratory have employed an exclusively alternating CS and US. In the present study, the effects of the alternating paradigm were compared with the effects of a pseudorandom schedule of CS and US presentations.

\section{METHOD}

\footnotetext{
Subjects

The experiment was conducted on 72 adult male and female cats weighing between 2.5 and $5.5 \mathrm{~kg}$. These animals were of unknown origin and were obtained from the animal care facility at Upstate Medical Center.
} 


\section{Surgical Procedure}

The animals were originally anesthetized with ether, a tracheotomy was performed, and a tube was inserted for artificial respiration. Ether anesthesia was discontinued and a mixture of $60 \%$ nitrous oxide, $38 \% \mathrm{O}_{2}$, and $2 \%$ halothane was administered through the respirator to maintain anesthesia. A carotid artery was cannulated for the measurement of blood pressure. Next, the spinal cord was transected between T-8 and T-10 with a thermal cautery. Care was taken not to injure blood vessels ventral to the cord. The animal's hindquarters were rigidly fixed with hip pins, and the left hindleg was fixed in a semi-flexed position (see Figure 1) by means of a foot clamp and a femur pin. The cutaneous superficial peroneal nerve and the cutaneous saphenous nerve were disected free at two locations. Bipolar stimulating and recording electrodes contacted the nerves as shown in Figure 1. Skin flaps were formed and all exposed nerve tissue was covered with warm mineral oil. The tendon of the tibialis anterior muscle of this leg was cut distally and attached to a Grass force transducer (resting tension about $60 \mathrm{~g}$ ). The force transducer leads were connected to a Grass polygraph where both low- and high-gain recordings of tension were made. The 72 animals were immobilized with one of two different lesions, which resulted in widely different mean arterial pressures: (1) Thirty-six of the animals were anemically decapitated by ligation of the carotid and vertebral arteries (anemic decapitate group). (2) The other 36 animals were decerebrated by a midcollicular transection using a thermal cautery (MCT group).

Subsequently, anesthesia was discontinued and the animal was allowed a minimum of $2 \mathrm{~h}$ to recover from the effects of spinal shock and anesthesia. A forelimb vein was cannulated for the administration of lactated Ringer's to maintain fluid volume, and a Beckman gas analyzer was used to monitor the $\mathrm{CO}_{2}$ concentration of the expired air. This concentration was maintained at $4.0 \%$ by adjusting the rate or volume of the respirator. A rectal thermometer was used to observe the cat's temperature, which was maintained between $36^{\circ}$ and $38^{\circ} \mathrm{C}$ by means of a hot-water heating pad.
Experiments on an additional six cats were not completed; three of these cats died before the end of the experiment and three others did not respond with flexion to saphenous nerve stimulation. All of these animals were immobilized by the anemic decapitation method.

\section{Procedure}

The CS was stimulation of the cutaneous saphenous nerve at an intensity which fired both $\mathrm{A} \alpha$ and Ad cutaneous fiber groups maximally but was below C fiber threshold (Hunt \& McIntyre, 1960, designation of cutaneous fiber types). The fiber type stimulated, and the constancy of this afferent input to the spinal cord for the CS, was monitored through the bipolar recording electrodes on a Tektronix dual-beam oscilloscope (Durkovic, 1975). A CS presentation consisted of a train of $0.2-\mathrm{msec}$ pulses delivered through a stimulus isolation unit by a Grass S88 stimulator. The train was $1.5 \mathrm{sec}$ long with a frequency of 10 pulses/sec.

The US was delivered to the superficial peroneal nerve in a similar manner and consisted of 30 pulses $/ \mathrm{sec}$ for $0.5 \mathrm{sec}$. Recordings from superficial peroneal nerve were used to monitor the fiber types activated by the US (Durkovic \& Light, 1975). Presence of $\mathrm{C}$ fiber activity induced by the US was confirmed at the end of appropriate experiments after a neuromuscular blocking agent (Flaxedil) was administered to prevent EMG from interfering with the nerve recording.

The animals were divided into three groups, each of which received a different US intensity. Group 1 received a US intensity which excited $70 \%-95 \%$ of the A $\alpha$ cutaneous fibers but no Ad fibers as determined by monitoring the compound action potentials. Group 2 received a US intensity that was sufficient to excite $\mathrm{A} \alpha$ and Ad fibers maximally, but did not excite $\mathrm{C}$ cutaneous fibers. Group 3 received a US intensity which excited all three cutaneous fiber groups ( $\mathrm{A} \alpha, \mathrm{A} \delta$, and $\mathrm{C}$ ) maximally.

Three stimulus presentation paradigms were used in this experiment. The first (the conditioning paradigm) consisted of CS presentations every minute (ITI $=60 \mathrm{sec}$ ), with the last $0.5 \mathrm{sec}$ of

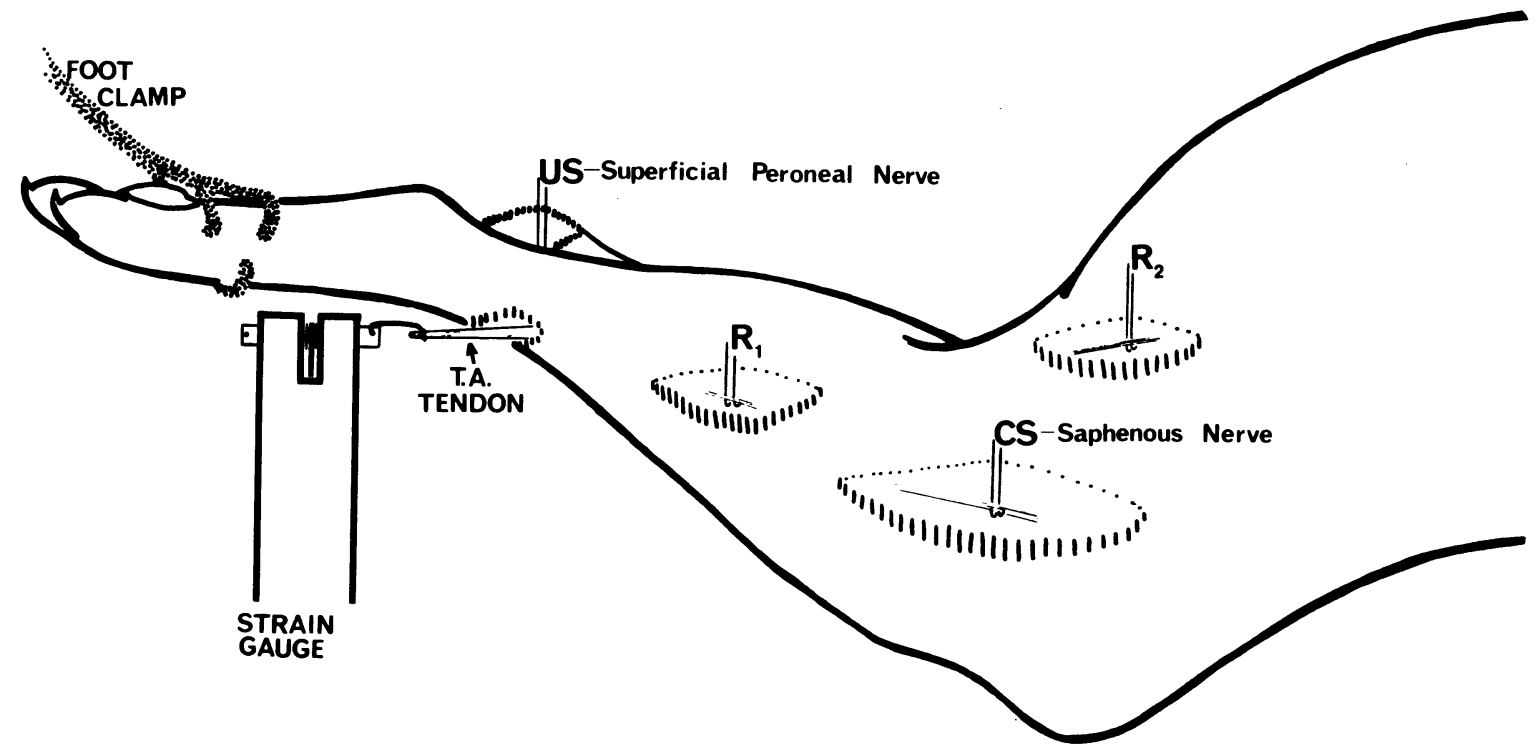

MEDIAL VIEW - LEFT LEG

Figure 1. Electrode and transducer placement. (US) Unconditioned stimulus is applied to the superficial peroneal cutaneous nerve, which is isolated near the position shown. (CS) Conditioned stimulus is applied to the saphenous cutaneous nerve. $R_{1}$ and $R_{2}$ are bipolar recording electrodes which monitor the fiber type stimulated and constancy of the afferent volleys. 
each CS being overlapped by the US. In the second (the alternating sensitization paradigm), CS and US were alternated, with presentations every $30 \mathrm{sec}$. The third (the pseudorandom sensitization paradigm) consisted of stimulus presentations every $30 \mathrm{sec}$, with CS or US being determined by a schedule which was random for every 10 stimulus presentations (5 CS and 5 US presentations). Thus, during the acquisition period, the sensitization animals received during each 5-min period, the same number of CS and US presentations as did the conditioning animals. However, the CS and US presentations were never paired for the sensitization animals.

The 72 animals were randomly assigned to the various groups until the levels of each factor were of equal number. Thus, Factor 1, the blood pressure factor, consisted of $36 \mathrm{MCT}$ animals and 36 anemic decapitate animals. Factor 2 , the stimulus presentation paradigm factor, contained 36 conditioning animals and 36 sensitization animals. The 36 sensitization animals were further divided so that there were 18 random and 18 alternating sensitization animals. Finally, Factor 3, the US-intensity factor, divided the 72 animals into three groups so that 24 animals received an A $\alpha$ US, 24 received an Aad US, and 24 received an A $a \delta C$ US. The experiment was, thus, designed for a 2 by 2 by 3 analysis of variance with a subdivision for the two different types of sensitization controls.

Following CS and US test stimuli (Durkovic, 1975) five CS alone trials were presented at 1 -min intervals to produce an initial, base-line flexion reflex of the tibialis anterior muscle. The response measure for every CS presentation was the peak (maximum) tension response during the first second of the CS. The mean of the five CS-alone trials was used as a baseline to which all subsequent five-trial mean peak tensions were compared.
One minute following the five CS-alone trials, 30 trials of CS and US presentations were administered, followed 1 min later by 15 more CS alone trials.

The experimental group means were compared by analysis of variance and Duncan's new multiple range test for acquisition and extinction periods, with $\alpha=0.05$ used for the significance level unless otherwise indicated.

\section{Results}

Blood pressure was measured before and after the experimental procedure. The mean of these readings was $75.6 \pm 2.5 \mathrm{SE} \mathrm{mmHg}$ for the anemic decapitate cats and $125.0 \pm 3.5 \mathrm{SE} \mathrm{mmHg}$ for the midbrain decerebrate animals.

The five initial CS-alone trials were used as the control, and all subsequent five-trial blocks were converted to a percentage using the mean of these first five trials as $100 \%$. The average tension, across all 72 animals, for these first five trials, was $396.5 \mathrm{~g}$. A 2 by 2 by 3 analysis of variance demonstrated that this base-line control was not significantly different for any of the experimental groups. This is important, because the data is expressed as percentages of these control levels. The data for the next 30 stimulus presentations (six trial blocks of five trials

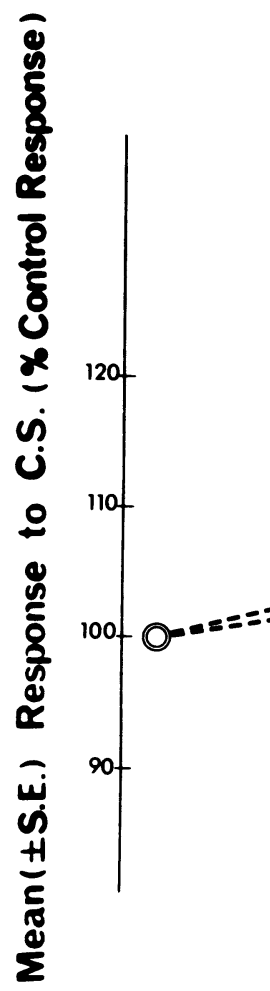

\section{Aa US}

Figure 2. A $\alpha$ US results. Trial blocks consist of five CS presentations. Open circles are sensitization means; closed circles, conditioned means. Each point represents the five-trial mean of the peak response to the CS \pm standard error (SE).
Conditioning

SENSITIZATION GROUP $(N=12)$

Extinction

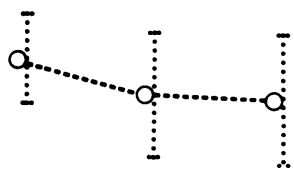

CONDITIONING

GROUP $(N=12)$

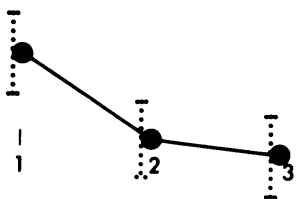

Trial Blocks 


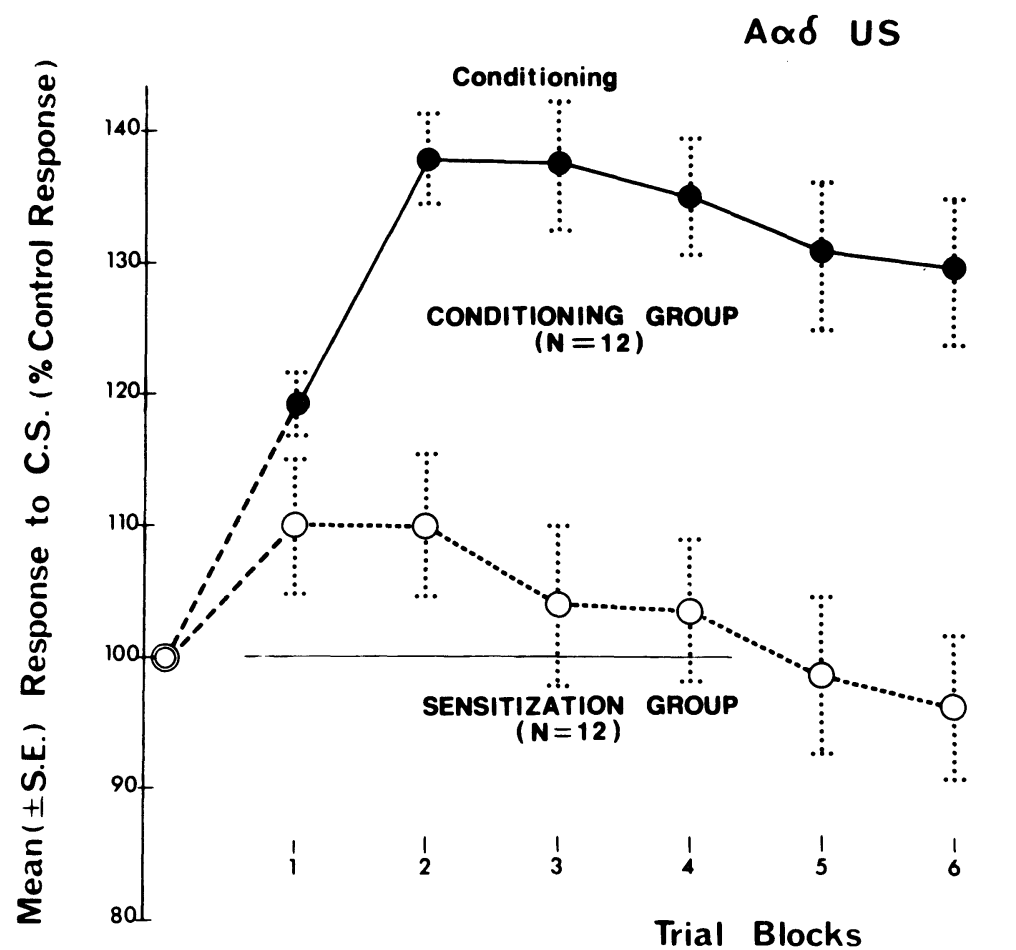

Extinction

Trial Blocks

Figure 3. A $\alpha \delta$ US results. Open circles, sensitization means; closed circles, conditioning means.

each) are shown in Figures 2, 3, and 4 for each of the US-intensity groups. For analysis, a single 30-trial mean for the acquisition period was computed for each animal. A 2 by 2 by 3 (Blood Pressure by Presentation Paradigm by US Intensity) analysis of variance was performed on these data. The results of this analysis of variance are shown in Table 1. This analysis demonstrated no significant differences in performance between the two blood-pressure groups $[F(1,60)=2.05, p>.1]$. As a result, bloodpressure groups were combined for the graphical representations.

Also, no significant difference was observed between the alternating or random sensitization groups $(p>.5)$, so these groups were combined for other analyses and graphs. The analysis of variance did demonstrate a significant main effect for Factor 2, stimulus presentation paradigm, during acquisition $[\mathrm{F}(1,60)=19.4, \mathrm{p}<.005]$. Also, the main effect of US intensity was significant $[F(2,60)=$ $8.23, \mathrm{p}<.005$ ], as was the first-order interaction between US intensity and stimulus presentation paradigm $[\mathrm{F}(2,60)=7.05, \mathrm{p}<.01]$.

Duncan's new multiple range test was used to test the simple main effects for the acquisition period, and demonstrated that $\mathrm{A} \alpha$ conditioning animals showed no greater reflex facilitation than did $\mathrm{A} \alpha$ sensitization animals (Figure 2). However, the condi- tioning animals in both the Aad (Figure 3) and the A $\alpha \delta C$ (Figure 4) groups exhibited significantly greater reflex facilitation than their respective sensitization controls $(\alpha<.001)$.

A comparison of the three US-intensity groups, using Duncan's new multiple range test, indicated that the $\mathrm{A} \alpha \delta$ and $\mathrm{A} \alpha \delta \mathrm{C}$ conditioning groups were significantly different from the $\mathrm{A} \alpha$ conditioning group $(\alpha<.001)$. The $\mathrm{A} \alpha \delta$ and $\mathrm{A} \alpha \delta \mathrm{C}$ conditioning groups did not differ from one another.

The three sensitization groups did not differ from one another on overall response during acquisition. However, a trend analysis of the five trial block data indicated that both the $\operatorname{A} \alpha \delta[\mathrm{F}(1,66)=7.7, \mathrm{p}<.025]$ and the $A \alpha \delta C[F(1,66)=20.0, p<.005]$ sensitization groups had significant quadratic trend components, while the A $\alpha$ group did not.

An analysis of variance was also performed on the extinction data (see Table 2). This analysis did not demonstrate any significant main effects. Only the first-order interaction between stimulus presentation paradigm and US intensity was significant. Reduced to simple effects, only the difference between $\mathrm{A} \alpha \delta \mathrm{C}$ conditioning and its sensitization control group was significant. Both $\mathrm{A} \alpha$ and $\mathrm{A} \alpha \delta$ conditioning groups were not significantly different from their sensitization control groups during extinction.

The UR was also recorded for all animals during the 


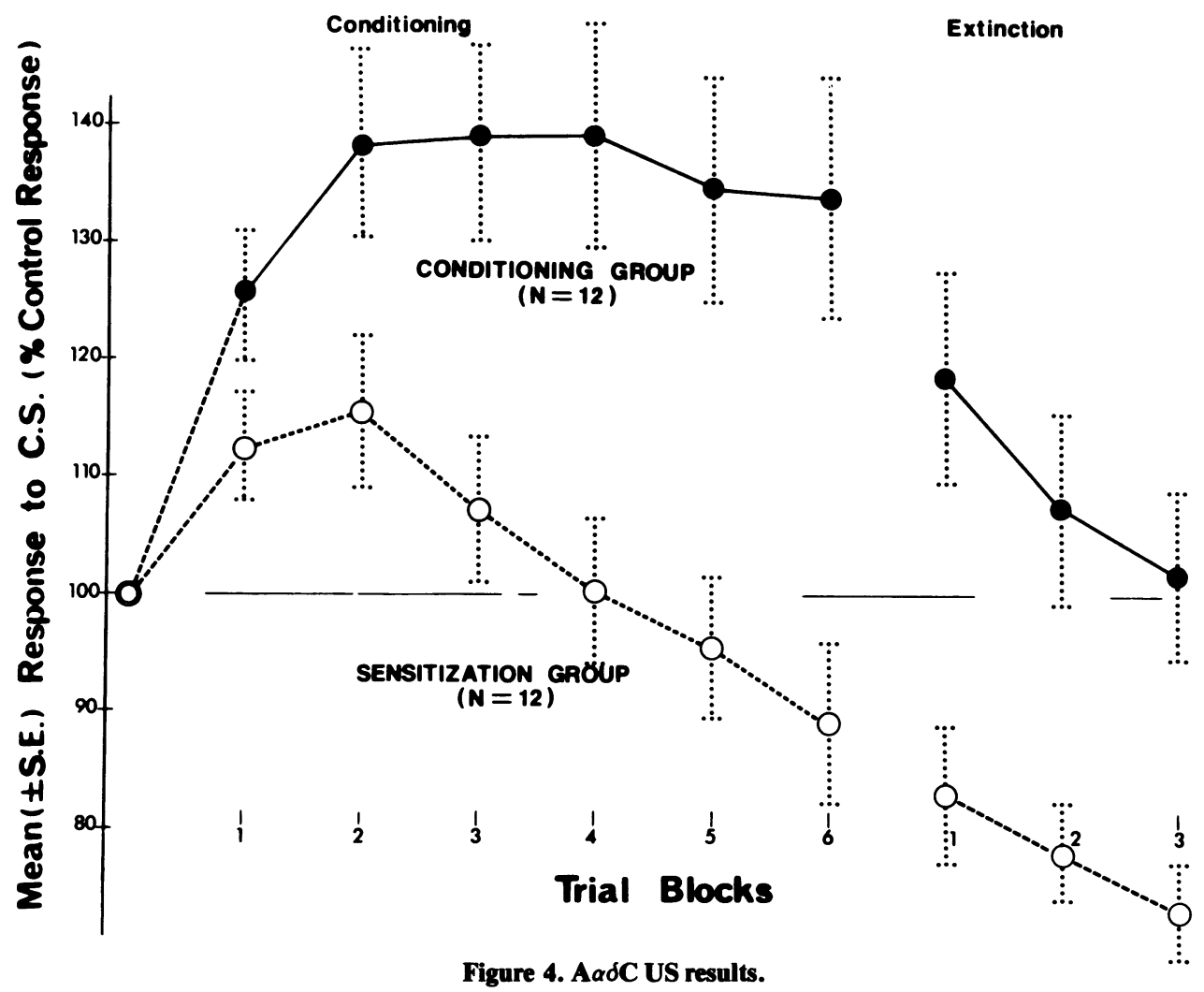

acquisition period. An analysis of variance was performed on the mean URs; the results are depicted in Table 3. This analysis demonstrated no effect of blood pressure on the UR $[F(1,60)=1.152, p>.1]$ and also no effect of stimulus presentations paradigm on the UR $[F(1,60)=1.82, p>.1]$. However, the effect of US intensity was significant $[F(2,60)=$ $10.67, \mathrm{p}<.005]$. As might be expected, the mean UR for the A $\alpha$ group (834.5 g) was significantly less than the UR for the Aad and A $\alpha \delta C$ groups $(\alpha<.01)$. The UR for the Aad group was $1,256.7 \mathrm{~g}$, which was not significantly less than the UR for the AadC group $(1,479.3 \mathrm{~g})$.

\section{DISCUSSION}

The most important finding of the present report is additional confirmation that the flexion reflex of the acute spinal cat is facilitated more during a classical conditioning paradigm than during sensitization procedures. Furthermore, this conditioned facilitation was found to be dependent on the activation by the US of A $\alpha \delta$ cutaneous nerve fibers. When the US recruited only $\mathrm{A} \alpha$ cutaneous fibers,
Table 1

Analysis of Variance: Acquisition

\begin{tabular}{lcrc}
\hline Source & df & \multicolumn{1}{c}{ MS } & \multicolumn{1}{c}{ F } \\
\hline Blood Pressure (A) & 1 & 665.39 & 2.05 \\
Stimulus Presentation Paradigm (B) & 1 & 6283.95 & $19.40^{* *}$ \\
US Intensity (C) & 2 & 2666.61 & $8.23 * *$ \\
A by B & 1 & 154.76 & .4777 \\
A by C & 2 & 292.67 & .9034 \\
B by C & 2 & 2284.56 & $7.05 *$ \\
A by B by C & 2 & 142.09 & .44 \\
\hline \multicolumn{1}{c}{${ }^{*} p<.01$} & & $*{ }^{*} p<.005$ &
\end{tabular}

Table 2

Analysis of Variance: Extinction

\begin{tabular}{lcrr}
\hline Source & df & \multicolumn{1}{c}{ MS } & F \\
\hline Blood Pressure (A) & 1 & 238.09 & .33 \\
Stimulus Presentation Paradigm (B) & 1 & 2541.49 & 3.53 \\
US Intensity (C) & 2 & 124.84 & .17 \\
A by B & 1 & 109.40 & .15 \\
A by C & 2 & 122.97 & .17 \\
B by C & 2 & 3486.74 & $4.84 *$ \\
A by B by C & 2 & 275.33 & .38 \\
\hline${ }^{*} p<.05$ & & &
\end{tabular}


Table 3

Analysis of Variance: UR Means

\begin{tabular}{lccc}
\hline Source & df & MS & F \\
\hline Blood Pressure (A) & 1 & $2.78 \times 10^{5}$ & 1.15 \\
Stimulus Presentation Paradigm (B) & 1 & $4.38 \times 10^{5}$ & 1.82 \\
US Intensity (C) & 2 & $25.74 \times 10^{5}$ & $10.67^{*}$ \\
A by B & 1 & 342.9 & .014 \\
A by C & 2 & $1.34 \times 10^{5}$ & .55 \\
B by C & 2 & $3.55 \times 10^{5}$ & 1.47 \\
A by B by C & 2 & $.30 \times 10^{5}$ & .13 \\
\hline
\end{tabular}

${ }^{*} p<.005$

no differences were observed between conditioning and sensitization control animals.

It should be noted that for these A $\alpha$ US groups, the CS (which activated both $\mathrm{A} \alpha$ and Ad fibers) could be looked upon as a more intense stimulus than the US. Pavlov (1927, pp. 30-31) noted that, in order for conditioning to occur, the CS must be a biologically less potent stimulus than the US. Lack of conditioning in the A $\alpha$ US group, then, could be viewed as experimental confirmation of Pavlov's observation at the spinal cord level.

However, if one uses the magnitude of the flexion reflex responses as an empirical method to estimate stimulus potency, such an interpretation of the results is no longer tenable. This is because the A $\alpha$ US produced approximately twice as large a maximum tension response than did the A $\alpha \delta$ CS (834.5 $\mathrm{g}$ for the mean UR vs. $366.0 \mathrm{~g}$ for the mean CS control response). ${ }^{1}$

An alternative way of interpreting these results is in the context of psychophysiological principles related to US intensity. In defense conditioning studies, a nociceptive, or painful, US intensity is apparently required to produce conditional responding in intact animals (see Durkovic \& Light, 1975). Cutaneous nerve stimulation studies have shown that Ad fibers must be activated in order for subjects to report pain (Burke, Mackenzie, Skuse, \& Lethlean, 1975; Collins, Nulsen, \& Randt, 1960; Heinbecker, Bishop \& O'Leary, 1933; Mackenzie, Burke, Skuse, \& Lethlean, 1975). Thus, the present study, which suggests the requirement of Ad fiber activation by the US for positive spinal conditioning results, corresponds to apparent US requirements for defense conditioning in intact animals.

Other characteristics which spinal conditioning shares with intact animal conditioning are: lack of backward conditioning (Patterson, 1975), optimal ISI of $250 \mathrm{msec}$ (Patterson, 1976), and decrease of conditioned facilitation to baseline levels during extinction (Durkovic, 1975; Durkovic \& Light, 1975; Fitzgerald \& Thompson, 1967; Patterson, 1975, 1967; Patterson et al., 1973). Thus, the spinal cord seems to be capable of mediating a response with many of the same parametric features of classical conditioning that have been observed in intact animals.

In the present study, no differences were observed between animals receiving random and alternating sensitization paradigms. These results make it unlikely that differences observed between conditioning and sensitization control animals are a result of the particular intertrial intervals employed.

Comparison of the present results with those of other similar studies do suggest that sensitization procedures produce somewhat different results, depending on whether or not the preparation is paralyzed. In the present study, both alternating and random sensitization groups exhibited some facilitation to the CS during the first 10 trials when an $\mathrm{A} \alpha \delta$ or $\mathrm{A} \alpha \delta \mathrm{C}$ US was used. This was indicated by the significant quadratic trend component observed in the data from these groups. Such facilitation during the first few trial blocks has not been observed in paralyzed cat preparations during unpaired CS and US presentations (Patterson et al., 1973) or during backward pairing (Patterson, 1975). Furthermore, in the paralyzed preparation, no differences were observed between sensitization and CS-alone control groups (Patterson et al., 1973), in contrast to the significant differences found between similar groups of nonparalyzed animals (Durkovic, 1975).

An explanation for at least some of the difference in sensitization effects between the paralyzed and unparalyzed spinal preparation may be an increased alpha motoneuron excitability in the unparalyzed preparation. It has been suggested that such an excitability increase may be brought about as a result of an increase in spontaneous discharge of muscle spindle afferent fibers from muscles involved in the flexion reflex (Durkovic, 1976). Since the production of changes in spindle afferent discharge brought about by cutaneous nerve stimulation would be blocked in the paralyzed preparation, there would be no increase in alpha motoneuron excitability from this source.

In the nonparalyzed preparation, effects from changes in muscle spindle afferent discharge would be expected to be similar in conditioning and sensitization control animals. These and other effects (e.g., fatigue) would be reflected as a change over time in the reactivity of the preparation to the CS. If one assumes that such effects are additive to the associative phenomena which produce increased responding during conditioning, one might obtain a first approximation of pure associative phenomena by subtracting sensitization results from the data obtained from conditioning animals (e.g., see Kling, 1971, p. 578). The results of such subtraction for each of the three US intensity groups are shown in Figure 5. These "net conditioning" curves illustrate that the 

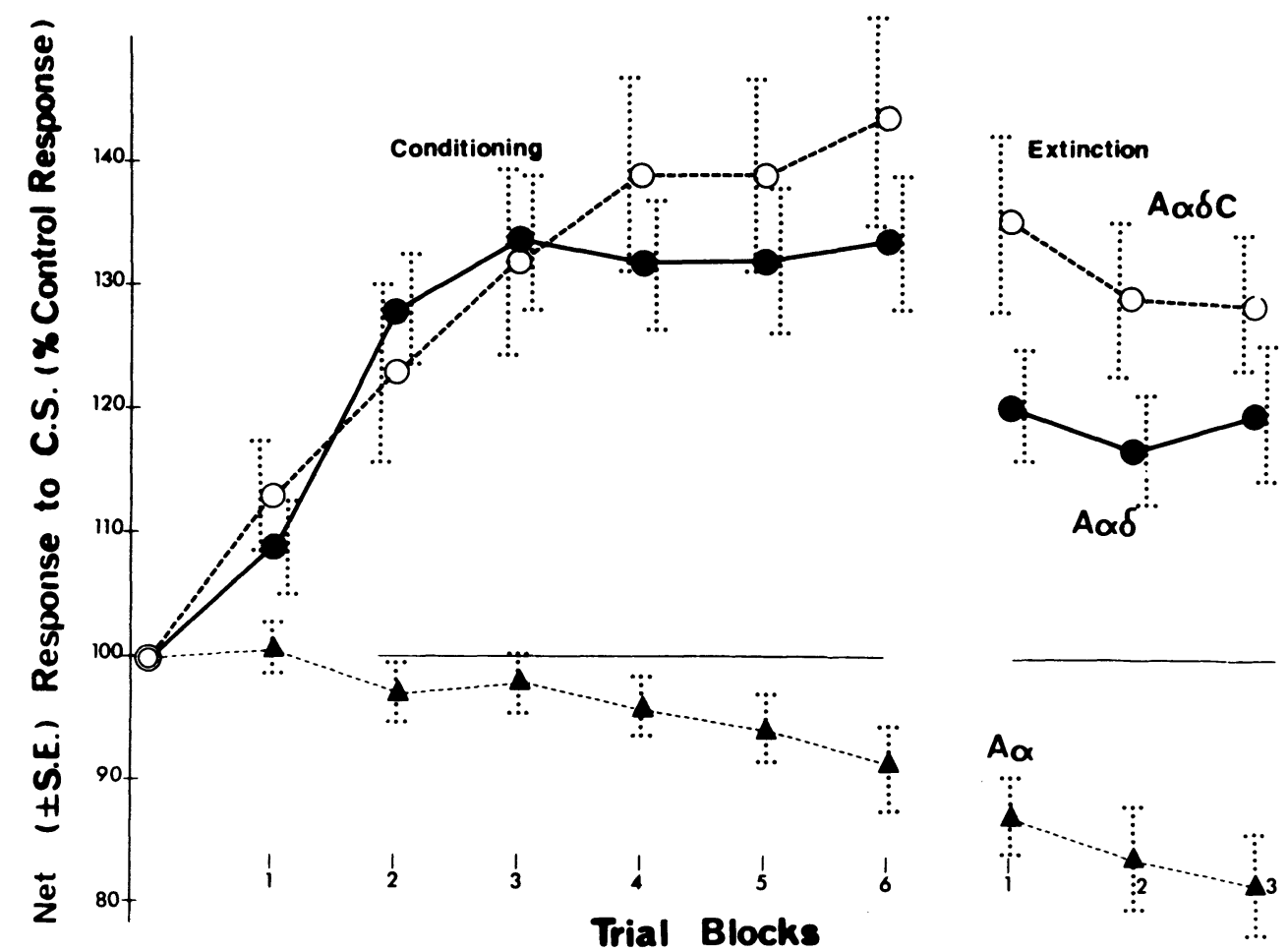

Figure 5. Net conditioning results. Points are computed by subtracting the sensitization means from the conditioning means and then adding $100 \%$. Open circles, AadC net results; closed circles, Aad net results; triangles, $A \alpha$ net results.

A $\alpha \delta$ and A $\alpha \delta C$ groups show very similar facilitation during classical conditioning procedures. In contrast, the A $\alpha$ US-intensity group remains at or below baseline during conditioning.

The results of this study also indicate that both the anemic decapitate and midbrain decerebrate spinal cat preparations demonstrate similar facilitation and variability during acquisition and extinction. This was somewhat surprising to us, since we had expected the MCT animals, with their higher blood pressure and therefore presumably better cord blood flow, to exhibit less interanimal response varibility. However, as was observed in a previous study using only anemic decapitate animals (Durkovic, 1975), the behavior of the two or three poorest MCT conditioning animals was indistinguishable from that of the MCT sensitization animals which demonstrated the greatest sensitization. A possible explanation of the similarity of results of high- and lowblood-pressure groups in this study is that, like dog (Griffiths, 1973) and monkey (Kobrine, Doyle, Newby, \& Rizzoli, 1976), the cat spinal cord may exhibit autoregulation of blood flow over these pressure ranges.

While it is unclear that classical conditioning at the spinal cord level has any significance in an intact animal's behavior, this phenomenon may still be important because of the potential use of the spinal cord as a simple model in which classical conditioning can be studied neurophysiologically. The advantage of the spinal cord as a learning model is its relative simplicity. Its afferent input can be easily controlled, the number of neural elements are relatively limited, it is amenable to neurophysiological techniques, and its neuroanatomy has been determined to a large extent. The present study has further simplified this model by excluding the necessity of $\mathrm{C}$ fiber activity in the production of classically conditioned flexion reflex facilitation. This reduces the number of spinal cord elements which must be studied and should make determinations of the involved reflex arcs easier. In addition, this study has demonstrated that wide range of systemic blood pressure are tolerated by the neural elements which are crucial for the conditioned behavior. This information shows that a conditioning preparation may be easily maintained within adequate limits during neurophysiological investigations. Thus, the spinal cord appears to be a particularly suitable mammalian model in which the neural basis of a classical conditioned response may be studied. 


\section{REFERENCES}

Burke, D., Mackenzie, R. A., Skuse, N. F., \& Lethlean, A. K. Cutaneous afferent activity in median and radial nerve fascicles: A microelectrode study. Journal of Neurology, Neurosurgery and Psychiatry, 1975, 38, 855-865.

Collins, W. F., Nulsen, F. E., \& Randt, C. T. Relation of peripheral nerve fiber size and sensation in man. Archives of Neurology (Chicago), 1960, 3, 381-385.

Durkovic, R. G. Classical conditioning sensitization and habituation in spinal cat. Physiology and Behavior, 1975, 14, 297-304.

Durkovic, R. G. Aftereffects of static or dynamic fusimotor activation on primary afferent discharge. Experimental Neurology, 1976, 50, 99-112.

Durkovic, R. G., \& Light, A. R. Spinal conditioning: Unconditioned stimulus intensity requirement. Brain Research, 1975, 98, 364-368.

Fitzgerald, L. A., \& Thompson, R. F. Classical conditioning of the hindlimb flexion reflex in the acute spinal cat. Psychonomic Science, 1967, 8, 213-214.

Griffiths, I. R. Spinal cord blood flow in dogs: The effect of blood pressure. Journal of Neurology, Neurosurgery and Psychiatry, 1973, 36, 914-920.

Heinbecker, P., Bishop, G. H., \& O'Leary, J. Pain and touch fibers in peripheral nerves. Archives of Neurology and Psychiatry (Chicago), 1933, 29, 771-789.

Hunt, C. C., \& McIntyre, A. K. An analysis of fibre diameter and receptor characteristics of myelinated cutaneous afferent fibres in cat. Journal of Physiology (London), 1960, 153, 99-112.

KLING, J. W. Learning. In J. W. Kling \& L. A. Riggs (Eds.), Experimental psychology. New York: Holt, Rinehart, \& Winston, 1971. Pp. 551-613.
Kobrine, A. I., Doyle, T. F., Newby, N., \& Rizzoli, H. V. Preserved autoregulation in the rhesus spinal cord after high cervical cord section. Journal of Neurosurgery, 1976, 44, 425-428.

Mackenzie, R. A., Burke, D., Skuse, N. F., \& Lethlean, A. K. Fibre function and perception during cutaneous nerve block. Journal of Neurology, Neurosurgery and Psychiatry, 1975, 38, 865-873.

Patterson, M. M. Effects of forward and backward classical conditioning procedures on a spinal cat hind-limb flexor nerve response. Physiological Psychology, 1975, 3, 86-91.

Patterson, M. M. Mechanisms of classical conditioning and fixation in spinal mammals. In A. Rieson \& R. F. Thompson (Eds.), Advances in psychobiology (Vol. III). New York: Wiley, 1976. Pp. 381.435.

Patterson, M. M. Cegavske, C. F., \& Thompson, R. F. Effects of a classical conditioning paradigm on hind-limb flexor nerve response in immobilized spinal cats. Journal of Comparative and Physiological Psychology, 1973, 84, 88-97.

Pavlov, I. P. Conditioned reflexes. London: Oxford, 1927. (Reprinted: New York: Dover, 1960)

\section{NOTE}

1. A combination of factors including differences in stimulus frequency, nerve size, and reflex capacity probably account for these differences in maximum tension produced by superficial peroneal and saphenous nerve stimulation.

(Received for publication March 3, 1976; revision accepted November $18,1976$. 